\title{
Constrained Jastrow calculations
}

DOI:

10.1016/0370-2693(75)90139-2

\section{Document Version}

Final published version

Link to publication record in Manchester Research Explorer

\section{Citation for published version (APA):}

Owen, J. C., Bishop, R. F., \& Irvine, J. M. (1975). Constrained Jastrow calculations. Physics Letters B, 59, 1-3. https://doi.org/10.1016/0370-2693(75)90139-2

\section{Published in:}

Physics Letters B

\section{Citing this paper}

Please note that where the full-text provided on Manchester Research Explorer is the Author Accepted Manuscript or Proof version this may differ from the final Published version. If citing, it is advised that you check and use the publisher's definitive version.

\section{General rights}

Copyright and moral rights for the publications made accessible in the Research Explorer are retained by the authors and/or other copyright owners and it is a condition of accessing publications that users recognise and abide by the legal requirements associated with these rights.

\section{Takedown policy}

If you believe that this document breaches copyright please refer to the University of Manchester's Takedown Procedures [http://man.ac.uk/04Y6Bo] or contact uml.scholarlycommunications@manchester.ac.uk providing relevant details, so we can investigate your claim.

\section{open $\partial_{\text {ACCESS }}$}




\title{
CONSTRAINED JASTROW CALCULATIONS
}

\author{
J.C. OWEN \\ Department of Theoretical Physics, University of Manchester, England \\ and \\ R.F. BISHOP and J.M. IRVINE \\ Department of Theoretical Physics, University of Manchester, England \\ and Daresbury Laboratory, Daresbury, Warrington, England
}

Received 11 August 1975

\begin{abstract}
We present an alternative to Pandharipande's lowest order constrained variational prescription for dense Fermi fluids, which is justified on both physical and strict variational grounds. Excellent results are obtained when applied to the "homework problem" of Bethe, in sharp contrast to those obtained from the Pandharipande prescription.
\end{abstract}

The desire to study such dense Fermi fluids as neutron star matter and liquid ${ }^{3} \mathrm{He}$ under pressure has led to renewed interest in variational techniques based on the use of Jastrow correlation functions [1]. The hope is that such techniques may prove more appropriate for microscopic calculations at high densities than the more familiar perturbation theory approach.

Several years ago Pandharipande [2] advanced a method of lowest order constrained variation (LOCV) which has subsequently been used in a number of studies of many-fermion systems. However, it has always been a problem to justify the Pandharipande technique on purely variational grounds. In this Letter we wish to propose an alternative approach to the problem which overcomes this difficulty.

In an attempt to compare different many-body techniques it has become the practice to test the various approaches against a simple idealized "homework problem". Upon the suggestion of Bethe this homework problem is a hypothetical system of neutrons interacting via the repulsive part of the Reid soft-core ${ }^{1} S_{0}$ potential (considered as a local potential acting in all partial waves),

$V(r)=6484.2 \mathrm{e}^{-7 x} / x \mathrm{MeV}$,

and $x=0.7 r$, with $r$ in fm. Furthermore, the neutrons are treated as Boltzmann particles, thus avoiding all the antisymmetrization complications due to spin-statistics. We shall demonstrate that our approach, applied to this "homework problem" gives results which are in excellent agreement with the essentially exact results of Shen and Woo [3] , and are significantly different from the predictions obtained using the method of Pandharipande.

A more detailed account of our approach, together with a fuller comparison with the LOCV of Pandharipande and results of calculations on a number of more realistic Fermi systems will be published elsewhere.

We consider an infinite system of spin-half fermions described by the Hamiltonian,

$H=-\frac{\hbar^{2}}{2 m} \sum_{i} \nabla_{i}^{2}+\sum_{i<j} V(i j)$.

With a trial wavefunction of the form,

$\Psi=\prod_{i<j} f(i j) \Phi$,

where $f(i j)$ is a two-body correlation function and $\Phi$ is a Slater determinant of uncorrelated single-particle states,

$\Phi=(N !)^{-1 / 2} \operatorname{det}\left\|\phi_{i}(j)\right\|$,

use of the variational principle

$E=\min \{\langle\Psi|H| \Psi\rangle\langle\langle\Psi \mid \Psi\rangle\}$,

provides us with an upper bound to the ground-state energy. This estimate for $E$ is expanded in a Van Kampen cluster series, 
$E=E_{1}+E_{2}+\ldots$,

where

$$
\begin{aligned}
& E_{1} / N=(3 / 10) \hbar^{2} k_{\mathrm{F}}^{2} / m, \\
& E_{2}=\sum_{i<j}\left(\phi_{i} \phi_{j} \mid \not \mathcal{A} f^{2}(12)\left\{-\hbar^{2} m^{-1}\left[\nabla_{1}^{2} f(12)\right.\right.\right. \\
& \left.\quad+\nabla_{1} f(12) \cdot \nabla_{1}+\nabla_{2} f(12) \cdot \nabla_{2}+V(12)\right\} \mathscr{A}\left|\phi_{i} \phi_{j}\right\rangle,
\end{aligned}
$$

and $A$ is the antisymmetrizing operator. It is our purpose to consider forms of the correlation function $f(12)$ for which truncation at the two-body cluster term will be valid.

In general the correlation functions are free to depend on both the relative linear momentum and angular momentum of the correlated pair. For the moment we shall ignore the momentum-dependence and display expressions only for the case where there are just two correlation functions $f_{t} ; t=1,2$, for odd and even partial waves respectively. In order to overcome the "Emery Difficulty" [4], the constraint

$0 \leqslant f_{t}(r) \leqslant 1$,

is imposed. Taking the continuum limit, and assuming

(i) $f_{t}(0)=0$, for repulsive potentials;

(ii) $f_{t}(r) \rightarrow 1, \quad r \rightarrow \infty$;

(iii) $f_{t}(r)$ and $f_{t}^{\prime}(r)$ are continuous;

we find,

$$
\begin{aligned}
& E_{2} / N=(6 \pi)^{-1} k_{\mathrm{F}} \sum_{t=1}^{2} \mathrm{~d} r\left\{\hbar^{2} m^{-1} f_{t}^{\prime 2}(r)\right. \\
& \left.+V_{t}(r) f_{t}^{2}(r)\right\} a_{t}^{2}\left(k_{\mathrm{F}} r\right),
\end{aligned}
$$

where

$a_{1}^{2}(x)=3\left[x^{2}-9 j_{1}^{2}(x)\right]$,

$a_{2}^{2}(x)=x^{2}+9 j_{1}^{2}(x)$,

with $j_{1}(x)$ a spherical Bessel function.

The dominant effect of our two-body correlation functions should be to keep any particle outside the range of the repulsive core of any other. We can restrict correlations to be amongst nearest neighbours on average, by imposing the condition that the average number of particles expelled by the correlations from some suitable core radius $R_{\mathrm{c}}$ drawn around any particle, be less than one. In the two-body approximation this implies,

$$
(3 \pi)^{-1} k_{\mathrm{F}} \sum_{t=1}^{2} \int_{0}^{R_{\mathrm{c}}} \mathrm{d} r\left[1-f_{t}^{2}(r)\right] a_{t}^{2}\left(k_{\mathrm{F}} r\right) \leqslant 1 .
$$

Since simultaneous imposition of the two constraints (8) and (11) forces $f_{t}(r)$ to heal rapidly to unity, we may replace $R_{\mathrm{c}} \rightarrow \infty$ with the effect of only slightly strengthening this constraint. The correlation functions are now functionally varied to minimize the expression (9) subject to the constraints (8) and (11). We thus have the two uncoupled Euler-Lagrange equations, each containing the same Lagrange multiplier, $\lambda$, due to constraint (11),

$$
\begin{aligned}
& g_{t}^{\prime \prime}(r)-\left\{a_{t}^{\prime \prime}\left(k_{\mathrm{F}} r\right) / a_{t}\left(k_{\mathrm{F}} r\right)\right. \\
& \left.\quad+m \hbar^{-2}\left[V_{t}(r)+\lambda\right]\right\} g_{t}(r)=0,
\end{aligned}
$$

where $g_{t}(r) \equiv a_{t}\left(k_{\mathrm{F}} r\right) f_{t}(r)$, and primes denote differentiation with respect to $r$. Eq. (12) has only one solution regular at the origin, but with an arbit:ary normalization $A_{t}$. Constraint (8) is imposed exactly by solving eq. (12) out to some distance $d_{t}$, and then setting $f_{t}(r) \equiv 1$ for $r>d_{t}$. The distance $d_{t}$ is chosen as large as possible with $f_{t}\left(r<d_{t}\right)<1$, and this clearly implies that $d_{t}$ is determined by $f_{t}^{\prime}\left(d_{t}\right)=0$. We then determine $A_{t}$ such that $f_{t}\left(d_{t}\right)=1$, and $f_{t}\left(r>d_{t}\right) \equiv 1$. The constant $\lambda$ is initially set to zero, and if the inequality (11) is not satisfied (now with $R_{\mathrm{c}} \rightarrow d_{t}$ by our solution), $\lambda$ is varied iteratively, solving for $f_{t}(r)$, $d_{t}$, and $A_{t}$ at each step until the constraint (11) is satisfied as an equality. For an account of the treatment of the parameters corresponding to $\lambda$ and $d_{t}$ in the Pandharipande prescription, see ref. [2] .

This procedure has been applied by us to the "homework problem" using the potential (1). The above expressions, all derived for the case of Fermi statistics, are simplified in this case, where we assume Boltzmann statistics, by replacing $\mathscr{A} \rightarrow 1$, which has the effect of replacing $j_{1}(x)$ by zero in eq. $(10)$ and enabling us to use just one correlation function to describe all partial waves. The results of Chakravarty, Miller and Woo (CMW) [5], Shen and Woo (SW) [3], and Pandharipande $(\mathrm{P})$ for this system have been compared in a recent paper by SW [3] , and in fig. 1 we add the present results to this compilation. The aforementioned groups all use different variational techniques, and all except $P$ involve the calculation of 


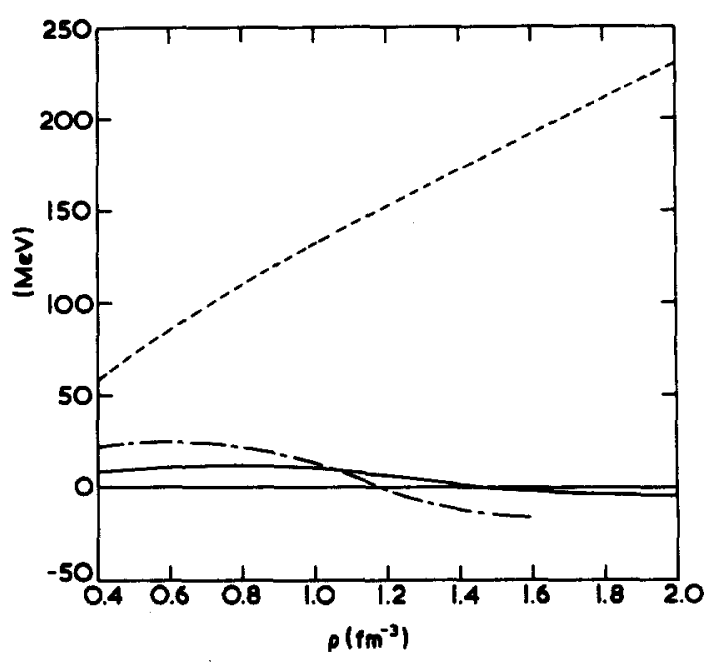

Fig. 1. Comparison of the results of several groups for the "homework problem" of Bethe (taken from fig. 1 of ref. [3]); $\left.\left.\left.-(E / N)_{\text {present }}-E / N\right)_{\mathrm{SW}} ; \cdots \cdot \ldots-\ldots / N\right)_{\mathrm{p}}-E / N\right)_{\mathrm{SW}}$ $\left.\cdots \cdots \cdot \ldots / N)_{\mathrm{CMW}}-E / N\right)_{\mathrm{SW}}$

many-body terms in the cluster expansion. In particular the SW results, employing Monte Carlo techniques to calculate the many-body integrals, may be considered as exact, and the deviation of the other results from theirs is plotted in fig. 1 .
The results obtained by our method are in excellent agreement with those of SW and clearly superior to those derived from the Pandharipande technique. At first sight it may appear strange that our results should agree so well with those of SW, especially at high densities, since we have made no explicit allowance for higher order clusters. The major contribution to the positive higher order terms arises when the correlation functions $f_{t}(r)$ differ appreciably from unity for $r \gtrsim r_{0}$, the unit radius. Should this occur, however, the constraint (11) then forces the two-body energy $E_{2}$ away from its minimum in a positive direction. Thus the effect of the constraint is not only to keep higher order terms small as compared with an unconstrained variational calculation, but also to include in $E_{2}$ a contribution arising from these terms.

We wish to thank H.B. Ghassib and M.R. Strayer for several helpful discussions.

\section{References}

[1] J.W. Clark and P. Westhaus, Phys. Rev. 141 (1966) 833.

[2] V.R. Pandharipande, Nucl. Phys. A178 (1971) 123.

[3] L. Shen and C.-W. Woo, Phys. Rev. D. 10 (1974) 371.

[4] V.J. Emery, Nucl. Phys. 6 (1958) 585.

[5] S. Chakravarty, M.D. Miller and C.-W. Woo, Nucl. Phys. A220 (1974) 233. 